\title{
A Unified Framework of Granular Computing
}

\author{
Yiyu Yao \\ Department of Computer Science, University of Regina, Regina \\ Saskatchewan, Canada S4S 0A2, E-mail: yyao@cs.uregina.ca
}

\begin{abstract}
In this chapter, we propose a unified framework for a holistic understanding of granular computing. It is developed based on three perspectives, namely, the philosophical, the methodological, and the computational perspectives. The three perspectives lead to structured thinking, structured problem solving, and structured information processing. We argue that the subject of the study of granular computing is a web of interacting granules representing a problem to be solved. From the web of granules, one can derive descriptions with multiple hierarchies (i.e., multiview) and multilevel granularity in each hierarchy.
\end{abstract}

Keywords: granular computing triangle, hierarchical structures, structured thinking, structured problem solving, structured information processing

\section{Introduction}

An early developing stage of a theory or a methodology is typically characterized by a diversity of many views, proposals, and models, but the lack of a unified framework. Ideas are scattered, fragmentary, and isolated, instead of forming an integrated whole. With extensive studies and better understanding, it is expected that a much smaller set of well accepted and dominant views will eventually converge. A challenge is how to speed up this process so that the theory can be effectively used by many more people.

As an emerging field of study, granular computing faces the same challenge. In the past few years we have witnessed a fast growing interest in this 
area $[3,15,22,23,30,56,57]$. On the one hand, many interpretations, models, paradigms, methodologies, techniques, and tools have been proposed and investigated $[4,29,31,32,33,35,39,41,42,46,47,48,49,50,60,61]$. On the other hand, there does not exist a commonly accepted definition nor a commonly agreed framework $[5,43,51,52,53,55]$. The lack of a conceptual framework may slow down the further development of granular computing and make it difficult for us to see and to exploit the universal applicability, flexibility, and effectiveness of granular computing. At this early stage, it may be impossible to define precisely without controversy what granular computing is, its scopes, its theories, and its methodologies [52]. Nevertheless, results from the existing studies suggest that we are making good progress towards a conceptual framework for granular computing [51, 52, 53, 55]. The main objective of this chapter is to examine the basic components of such a framework.

From the existing studies, we can observe several limitations and problems. Many studies focus on specific issues, concrete models, and domainspecific methodologies. The existing studies are dominated by computational intelligence theories, including fuzzy sets, rough sets, neural networks, interval computing, and many more. It is also not surprising to find that some studies are simply reformulations of existing results by using the terminologies of granular computing without the necessary new insight. A conceptual framework of granular computing would enable us to avoid such problems. Studies of granular computing must be pursued in depth and results from those studies be integrated in breadth. Granular computing needs to be an interdisciplinary study related to many branches of science, moving away from the current domination of fuzzy sets and rough sets. It is also necessary to investigate distinguishing properties that justify granular computing as a separate field of study in its own right.

The basic principles and ideas of granular computing have, in fact, long appeared in many branches of science and many fields of computer science [51, $52,53,55]$. Unfortunately, they are scattered over many places in isolation and are not readily accessible, as they are either described and discussed under different names or buried in domain-specific details. Yet, those effective ideas and principles lend themselves immediately for a conceptual framework of granular computing. Two tasks are involved in building this framework. One is to extract high-level commonalities of different disciplines and to synthesize their results into an integrated whole by ignoring low-level details. The other is to make explicit ideas hidden in discipline-specific discussions 
in order to arrive at a set of discipline-independent principles.

In our view, granular computing is a new field of study that has emerged from many different disciplines and fields, including general systems theory $[7,8,20]$, hierarchy theory $[1,27,36,37,44]$, social networks $[2,8,16]$, artificial intelligence $[12,13,14,18,58]$, human problem solving [26], learning [10, 34], programming [11, 19, 21, 45], theory of computation [38], and information processing $[17,24]$. Although granular computing draws heavily upon results from other fields, it has unique and distinguishing characteristics. The proposed framework of granular computing is based on three related perspectives [54]. From the philosophical perspective, granular computing offers a new world view that leads to structured thinking. From the methodological perspective, granular computing deals with structured problem solving. From the computational perspective, granular computing concerns structured information processing. The integration of the three perspectives results in a holistic understanding of granular computing that emphasizes structures embedded in a web of granules.

The main aim of the framework is to bring a clear understanding of granular computing. The framework may not be completely accurate and many of its components and views may have to be refined with time. Although it is not certain if every perspective of the framework will be accepted eventually, there is no doubt that the study of a unified framework will play a crucial role in the development of a full theory of granular computing.

\section{Philosophical Perspective: Structured Thinking}

The philosophical view of granular computing may have a great impact on the current research in the field. Such a philosophical foundation, however, has hardly been examined. Although it may be too early to pinpoint this philosophical view, we can at least discuss and elaborate on some of its important features $[51,52,53,55]$. We believe that the philosophy of granular computing is structured thinking, characterized by hierarchical modeling, understanding, processing and learning. Those hierarchical processing tasks

are essential to human intelligence $[10,13,34,40]$. They have significant implications for knowledge-intensive information systems.

To put granular computing in its right perspective, we need first to briefly 
mention two complementary philosophical views dealing with the complexity of real world problems, namely, the traditional reductionist thinking and the new systems thinking. According to reductionist thinking, a complex system or problem can be divided into simpler and more fundamental parts, and they can be further divided. An understanding of the system can be reduced to the understanding of its parts. In other words, we can deduce fully the properties of the system based solely on the properties of its parts. In contrast, systems thinking shifts from parts to the whole, in terms of connectedness, relationships, and context [7, 8, 20]. A complex system is viewed as an integrated whole consisting of a web of interconnected, interacting, and highly organized parts. The properties of the whole are not present in any of its parts, but emerge from the interactions and relationships of the parts.

The reductionist thinking and systems thinking are considered by many as competing views. Since each of them is effective in modeling and solving different types of problems, we consider the two as complementary views. The existing research on granular computing is mainly influenced by the reductionist view. For example, phrases representing reductionist thinking, such as "divide and conquer" and "granulate and conquer", are used to explain the workings of granular computing. This bias may be corrected if granular computing can also draw results from systems thinking.

The reductionist thinking and systems thinking agree on the modeling of a complex system in terms of whole and parts, but differ in how to make inference with the parts. The two views exploit a common structure known as the hierarchical structure characterized by multiple levels. According to reductionist thinking, a system can be continually divided into smaller and smaller parts to form a multilevel hierarchial representation and understanding. In systems thinking, one can form different systems levels so that systems can be nested within other systems. Based on this common hierarchical structure, granular computing attempts to unify reductionist thinking and systems thinking.

Hierarchical structures and organizations exist in the real world, or more precisely, our perception of the real world. They can be found in many natural, social, and man-made systems [16, 27, 37, 44]. Humans have evolved to deal with such hierarchial structures effectively and efficiently [13, 14, 26, 37, 56]. Human perception and understanding of the world depends, to a large extent, on nested and hierarchical structures. We view and represent the world using various grain sizes and abstract only those things that serve the present interests. The ability to conceptualize the world at different levels of 
granularity and to switch among these levels is fundamental to human intelligence and flexibility [14]. It is also interesting to comment that hierarchical structures have been used by some authors to explain the human brain and intelligence. For example, Hawkins [13] proposes that the human brain can be conceptually understood by a cortical hierarchy model that mirrors the hierarchical structures of the real world.

The notion of hierarchical structures captures the essential features of our perception and understanding of the world at multiple levels of granularity [14, 29, 56]. Granular computing, formulated based on hierarchical structures, promotes a way of structured thinking by combining analytic and synthetic methods. Analytic thinking involves the division of a whole into relatively independent parts. This allows us to move to a lower level in a hierarchical structure where individual properties of parts can be studied. On the other hand, synthetic thinking enables us to combine parts into a complex whole. This enables us to move to a higher level in a hierarchical structure where the emergent properties of the whole can be examined.

\section{Methodological Perspective: Structured Problem Solving}

The philosophical foundations of granular computing is a view of the world in terms of granules and multiple levels of granularity. In search of methods of problem solving, this hierarchical structure plays a crucial role. From the methodological perspective, granular computing is structured problem solving guided by the structured thinking. By drawing results from structured programming, artificial intelligence, hierarchy theory, rough set theory [28], quotient space theory $[58,59]$, and others, one may extract a set of fundamental principles for systematic problem solving [51, 52, 53, 54, 55].

The philosophy of granular computing implies two mutually dependent tasks of structured problem solving, namely, constructing a hierarchical view and working with the associated hierarchy. In some cases, the separation of the two tasks is not clear. It may happen that the two tasks are tied together instead of one following the other. Many principles can be applied to both tasks. As examples, we examine three such principles.

A fundamental principle of granular computing is "the principle of multilevel granularity." This principle stresses the importance of breaking a 
large problem into smaller problems and understanding a problem at many levels of detail. One may construct many hierarchical views and select the most suitable view. A level-wise construction process can be done in either a top-down or a bottom-up manner, based on the properties of loose coupling of parts and near-decomposability [37]. A top-down level-wise construction process is consistent with the breadth-first search strategy of artificial intelligence. Alternatively, one may construct a hierarchical view based on the depth-first search strategy. Once a hierarchical view is created, working with a hierarchy is natural at multiple levels of granularity.

The principle of multilevel granularity can in fact be applied to the problem of constructing a hierarchical view. One may consecutively build different versions of a hierarchy with differing details. For example, a level in one version may be divided into two or more levels in the next version. Those different versions reflect naturally our multiple understandings of the problem.

Another principle of granular computing is "the principle of focused effort." This principle states that, at a given stage of constructing a hierarchy and working with the hierarchy, effort is to be concentrated on a particular granule or a specific level, relatively independent of other granules or levels. In doing so, one abstracts only those things that are relevant to the present interests and ignores irrelevant lower-level details or relationships to other things. The principle does not rule out the needs for some effort to be made on the study of related things. It requires the concentration of the major effort on a part, instead of the whole, at a specific point in time. The applications of this principle result in a concrete sequence of steps towards a complete and structured solution to a problem.

The third principle of granular computing is "the principle of granularity conversion." It calls for an easy switch between levels of abstraction. According to this principle, a hierarchy describing a problem should be constructed in a way to facilitate easy granularity conversion. When working with a hierarchy, one can fluently switch levels of granularity as well as passing information between levels.

The top-down analytic methods may be helpful in switching from a higher level to a lower level. By analysis, a large granule is broken into smaller granules. A solution with respect to larger granules can be derived by combining solutions from the corresponding families of smaller granules. In contrast, the bottom-up synthetic methods may be used for switching from a lower level to a higher level. By synthesis, the connections and interactions of 
lower-level granules may be studied and integrated. This may reveal emergent properties, the properties that none of the lower-level granules has, at the higher level.

The three principles are not new and have been either explicitly or implicitly used in many fields. For example, they can be easily seen from the principles of structured programming, although they are stated differently $[11,19,45]$. Our main objective is to demonstrate that, from the methodological perspective, granular computing is structured problem solving based on principles proven to be effective across different disciplines. Many other principles can be similarly reinterpreted in the light of granular computing. Collecting and presenting coherently those principles remain a great challenge.

\section{Computational Perspective: Structured Information Processing}

From the computational perspective, granular computing is structured information processing. It is about the applications of the granular computing philosophy and principles in the design and implementation of intelligent information systems. Our exploration of the computational perspective is based on two studies: the pyramid approach suggested by Bargiela and Pedrycz [3] for granular computing and the multiple levels approach proposed by Marr [24] for human and computer vision.

In the information processing paradigm proposed by Bargiela and Pedrycz [3], granular computing works with a pyramid consisting of levels of differentsized information granules, i.e., a hierarchical structure. The processing at different levels of information granulation is a necessary feature of any knowledge-intensive system. In the study of human representation and processing of visual information, Marr [24] makes a convincing argument for a multilevel understanding of an information processing system. At different levels of description, one explores different kinds of explanations. A coherent explanation, hopefully, may be obtained from explanations at those linked levels. The philosophical views and basic working principles, though presented with reference to information processing, are indeed much similar to ones of granular computing we discussed earlier.

For the explanation of specific information processing mechanisms and 
systems, Marr [24] uses the two notions of representation and process. A representation is a formal system that makes explicit certain entities or types of information and a specification of how the system does this. The result of using a representation to describe an entity is called a description of the entity in the representation. A process may be simply interpreted as actions or procedures for carrying out information processing tasks. It may also be interpreted as a mapping from one representation to another representation. In general, a representation may determine the effectiveness of processes under the representation. It may be necessary to choose a set of the most appropriate processes.

It is easy to describe the computational perspective on granular computing based on representation and process. As a minimum requirement, a representation of granules must capture essential features of granules and make a particular aspect of their physical meanings explicit. Furthermore, the representation of granules needs to be connected closely to representations of granular structures with respect to granules, levels, and hierarchies. In some cases, it may be possible to derive a representation of granular structures from the representation of granules.

Processes of granular computing may be broadly divided into the two classes of granulation and computation with granules [48, 54]. Granulation involves the construction of the building blocks and structures, namely, granules, levels, and hierarchies. Many issues are involved in granulation, including granulation criteria, granulation algorithms, and characterization of both granules and granular structures. Computation processes systematically explore the granular structures. This involves two-way communications up and down in a hierarchy, as well as switching between levels. For those tasks, we can define mappings connecting granules and levels, modes of granularity conversion, and operators of computing. For the consistency of computation at different levels, we need to study the issues of consistency preservation in terms of invariant properties.

Computation at a certain level may produce an approximate, a partial, or a schematic solution. Such a solution may be made more precise, more complete or more detailed at another level. This suggests that granular computing is a stepwise refinement process that has been successfully applied in structured programming [45].

Information processing at multiple levels serves the practical needs of an approximate solution within a tolerance range in order to gain in efficiency, low costs, or understandability. This trade-off is essential for solving many 
real-world problems. The hierarchical way of problem solving makes it easy to find the right level of approximation.

\section{$5 \quad$ A Unified Framework}

Based on the three perspectives, we are ready to develop a unified framework of granular computing and to consider its specific issues. A real-world problem consists of a web of interacting and interrelated granules. For effective problem solving, granular computing must extract easily-understandable structures to approximately represent the problem. This results in an understanding in terms of multiple hierarchies and multiple levels in each hierarchy.

\subsection{The Granular Computing Triangle}

In the earlier papers $[51,52,53,54,55]$, we suggest a linear dependency between the three perspectives. The philosophy of granular computing guides its methodologies and the methodologies are used to implement information processing systems. A further investigation convinced us that such an ordering may be inappropriate and perhaps misleading. The three distinctive perspectives mutually depend on each other. This new understanding leads to a trinity view of granular computing.

The three perspectives are distinct because they deal with issues of different categories and nature. Since each perspective can be further divided, one may study the divisions again from the three perspectives. The three perspectives are thus interrelated and form an integrated whole. Instead of putting them in a linear ordering, we can represent them as three points in a triangle called the granular computing triangle. In this way, any perspective is related to the other two.

In the unified trinity framework of granular computing, the three perspectives are tied closely together by their common focus on granular structures. The exploration of structures defined by multilevel granularity makes granular computing a promising field of study. The three perspectives represent the basic angles of such an exploration. This suggests another interpretation of the three perspectives. If they are understood as a basis of a threedimensional space, each point in the space represents a type of study with a specific emphasis on the three perspectives. 
The three-dimensional space interpretation enables us to review and compare the existing studies. It is not surprising to observe that the majority of studies have a strong bias to methodological and computational perspectives, and even more so to the computational perspective. The unified trinity framework stresses the fact that each perspective contributes significantly to the understanding of granular computing. None of them can be overlooked.

The unified conceptual framework considers more abstract disciplineindependent principles. It stresses the flexibility and universal applicability of granular computing. As a consequence, granular computing is applicable to solve a wide range of problems. For example, we may apply the principles of granular computing to the study of the subject of "granular computing." The result is a multilevel and multiview understanding. Our elaboration and description of granular computing in this chapter is, in fact, one such example.

\subsection{The Web of Granules}

A basic task of granular computing is to build a hierarchical model for a complex system or problem. The basic ingredients of granular computing are granules, a web of granules, and granular structures.

\subsubsection{Granules}

A complex problem consists of interconnected and interacting parts. Each part, in turn, consists of other parts. Intuitively, each part or a group of parts may be considered as a granule. We therefore have a web of granules as a representation of the problem under consideration. While granules provide local descriptions, the web of granules gives a complete picture of the problem.

We treat granules as a primitive notion of granular computing. From it, other notions can be derived. Furthermore, granules are an abstract notion. The physical meaning of granules can be made clear only when a particular application is considered. In modeling complex systems, granules may be the components of systems at different levels $[7,8]$. In programming, granules may be various modules of a software system [21]. In the theory of small groups, granules are small groups [2]. In any organization, granules may be various divisions and departments at different levels. Abstracting from all those concrete examples, a granule can be considered as a focal point of our 
interest at a certain stage in problem solving. Granules may correspond to either real world objects or their abstractions.

A granule can be either simple or compound. A simple granule cannot be further decomposed into, or formed by, other granules. A compound granule consists of a group of its interconnected and interacting element granules (they, in turn, may be simple or compound). A granule is related to other granules by its dual roles. A granule can be considered as a whole when it is viewed as a part of another granule. A granule is considered to be a group of interconnected and interacting granules when some other granules are viewed as its parts. Consequently, we need to characterize granules by a minimum set of three types of properties. The internal properties of a granule reflect its organizational structures, the relationships, and interaction of its element granules. The external properties of a granule reveal its interaction with other granules. The emergent properties of the granule may be viewed as one type of external property. In many cases, both the internal and external properties are not static but change with its environment. The contextual properties of a granule show its relative existence in a particular environment. The three types of properties together provide us a full understanding of the notion of a granule.

\subsubsection{Granular structures}

A complex problem is represented as a web of granules, in which a granule itself may be a web of smaller granules. With such a representation, an important issue is to study various granular structures embedded in this web. Those structures are crucial to an understanding of the problem at different levels.

The types of granular structures are related to the earlier discussed roles of granules and properties of granules. We consider three levels of granular structures $[53,55]$. The granule structures represent the internal structures of a compound granule. The structures are determined by its element granules in terms of their composition, organization, and relationships. Each element granule is considered simply as a single point when studying the structure of a compound granule. From the view point of element granules, the internal structures of a compound granule are indeed their collective structures. In general, one can study the collective structures of a family of granules. Each granule in the family captures and represents a particular and local aspect of the problem. Collectively, they represent the entire problem at a level 
of granularity defined by the granules in the family. One may use many families of granules to examine the problem at multiple levels of granularity. Structurally, the multiple levels form a hierarchy.

\subsection{Multilevel and Multiview}

With the introduction of granular structures, a problem is understood in terms of granules, levels, and hierarchies. Specifically, a level is made up of a family of granules and a hierarchy is made up of multiple such levels. This not only makes a complex problem more easily understandable, but also leads to efficient, although perhaps approximate, solutions.

Our discussion so far is based on the assumption that a complex problem can be expressed as a web of granules. A fundamental question regarding how those granules are formulated in the first place is not discussed. In fact, a complex problem in the real world is a web in which everything is connected to everything else [7]. The formation of granules is related to the notion of approximations and loose coupling of parts $[7,37]$. In forming a granule, one may ignore the subtle differences between its elements and between their individual connections to others. That is, a group of elements may be treated approximately as a whole when studying their relations to others. Each granule is a focal point of our investigation. As an example, the study of cluster analysis in fact relies on such granulated views. The knowledge obtained based on granules, although approximate, may be good enough for practical uses.

In building a hierarchical structure, we need to have a vertical separation of levels and a horizontal separation of granules at the same hierarchical level. Like the formation of individual granules, those separations explore the property of loose coupling of parts. The multiple hierarchical structure thus provides a practical model of a nearly-decomposable problem. The relationship between levels can be interpreted in terms of abstraction, control, complexity, detail, resolution, and so on.

Granular computing searches for a multilevel hierarchical view of a problem based on near-decomposability. Some useful information may be lost with such a hierarchy instead of a web. However, we gain in a model that is easier to understand, tractable, and economic.

A hierarchy represents the results of a study of a problem from one particular angle or point-of-view. For the same problem, many interpretations and descriptions may co-exist $[6,9]$. It may be necessary to construct and 
compare multiple hierarchies [16]. A comparative study of those hierarchies may provide a complete understanding of the problem.

The conceptualization of a problem through multiple hierarchies (i.e., multiview) and multilevel in each hierarchy is general and flexible. Such an approach has been widely used in the investigations of many branches of science. Each hierarchy represents one view of the problem. One may either focus on a particular view or compare various views. The latter requires the connections between different views. More importantly, emergent properties of a family of views may be observed, which are absent in any specific view.

\section{Concluding Remarks}

Our study of granular computing can be considered to be both old and new. It is old in the sense that the basic ideas and principles of granular computing have appeared time and again in many branches of science and fields of computer science. It is new in the sense that we attempt to extract a set of abstract discipline-independent ideas and principles, and in many cases make them explicit, to arrive at a unified trinity framework of granular computing.

The framework is based on three perspectives. The philosophical perspective focuses on structured thinking, the methodological perspective on structured problem solving, and the computational perspective on structured information processing. The exploration of hierarchical structures at multilevel granularity is the foundation of granular computing.

The field of granular computing is taking shape and will be immensely important. Its success can be predicted from the following observations. Scientists working at different disciplines deal with different subject matters. However, their research processes and methodologies are remarkably similar at a higher level [25]. What distinguishes scientists is their ways of thinking, rather than the subject matter. In general, human problem solving methodologies and skills share high-level similarities, independent of the problems being solved. The unified framework of granular computing is based on the extraction of such high-level ideas and principles. Although specific components may be refined or even be entirely changed, this framework may guide research of granular computing in the right direction.

In this article, we focus on a high-level examination of granular computing as a new field of study in its own right. The basic ideas and principles are

discussed in more abstract terms. Instead of giving more detailed examples 
to illustrate them, we provide an extensive list of references. One can easily find detailed discussions about the specific applications of these ideas and principles from the references. This high-level investigation enables us to understand granular computing as a new theory for its full potential power without distracted by minute details. The next logical steps, and a real challenge, are to study granular computing at lower levels, with many examples to illustrate its basic ideas and principles.

\section{References}

[1] V. Ahl, T.F.H. Allen, Hierarchy Theory, a Vision, Vocabulary and Epistemology, Columbia University Press, New York, 1996.

[2] H. Arrow, J.E. McGrath, J.L. Berdahl, Small Groups as Complex Systems: Formation, Coordination, Development, and Applications, Sage Publications, Thousand Oaks, California, 2000.

[3] A. Bargiela, W. Pedrycz, Granular Computing: An Introduction, Kluwer Academic Publishers, Boston, 2002.

[4] A. Bargiela, W. Pedrycz, Granular mappings, IEEE Transactions on Systems, Man, and Cybernetics, Part A 35 (2005) 292-297.

[5] A. Bargiela, W. Pedrycz, The roots of granular computing, Proc. 2006 IEEE Int. Conf. on Granular Computing, 2006, pp. 806-809.

[6] G. Bateson, Mind and Nature: A Necessary Unit, E.P. Button, New York, 1979.

[7] F. Capra, The Web of Life, Anchor Books, New York, 1997.

[8] F. Capra, The Hidden Connections: A Science for Sustainable Living, Anchor Books, New York, 2002.

[9] Y.H. Chen, Y.Y. Yao, Multiview intelligent data analysis based on granular computing, Proc. 2006 IEEE Int. Conf. on Granular Computing, 2006, pp. 281-286.

[10] C.M. Conway, M.H. Christiansen, Sequential learning in non-human primates, Trends in Cognitive Sciences 12 (2001) 539-546. 
[11] O.-J. Dahl, E.W. Dijkstra, C.A.R. Hoare, Structured Programming, Academic Press, New York, 1972.

[12] F. Giunchglia, T. Walsh, A theory of abstraction, Artificial Intelligence 56 (1992) 323-390.

[13] J. Hawkins, S. Blakeslee, On Intelligence, Henry Holt and Company, New York, 2004.

[14] J.R. Hobbs, Granularity, Proc. 9th Int. Joint Conf. on Artificial Intelligence, 1985, pp. 432-435.

[15] M. Inuiguchi, S. Hirano, S. Tsumoto (Eds.) Rough Set Theory and Granular Computing, Springer, Berlin, 2003.

[16] V. Jeffries, H.E. Ransford, Social Stratification: A Multiple Hierarchy Approach, Allyn and Bacon Inc., Boston, 1980.

[17] D. Klahr, K. Kotovsky (Eds.) Complex Information Processing: The Impact of Herbert A. Simon, Lawrence Erlbaum Associates, Hillsdale, New Jersey, 1989.

[18] C.A. Knoblock, Generating Abstraction Hierarchies: An Automated Approach to Reducing Search in Planning, Kluwer Academic Publishers, Boston, 1993.

[19] D.E. Knuth, Structured programming with go to statements, Computing Surveys 6 (1974) 261-301.

[20] E. Laszlo, The Systems View of the World: The Natural Philosophy of the New Developments in the Science, George Brasiller, New York, 1972 .

[21] H.F. Ledgard, J.F. Gueras, P.A. Nagin, PASCAL with Style: Programming Proverbs, Hayden Book Company, Rechelle Park, New Jersey, 1979 .

[22] T.Y. Lin, Granular computing, Announcement of the BISC Special Interest Group on Granular Computing, 1997.

[23] T.Y. Lin, Y.Y. Yao, L.A. Zadeh (Eds.) Data Mining, Rough Sets and Granular Computing, Physica-Verlag, Heidelberg, 2002. 
[24] D. Marr, Vision, a Computational Investigation into Human Representation and Processing of Visual Information, W.H. Freeman and Company, San Francisco, 1982.

[25] R.C. Martella, R. Nelson, N.E.Marchard-Martella, Research Methods: Learning to Become a Critical Research Consumer, Allyn and Bacon, Boston, 1999.

[26] A. Newell, H.A. Simon, Human Problem Solving, Prentice-Hall, Englewood Cliffs, New Jersey, 1972.

[27] H.H. Pattee, (Ed.) Hierarchy Theory, the Challenge of Complex Systems, George Braziller, New York, 1973.

[28] Z. Pawlak, Rough Sets, Theoretical Aspects of Reasoning about Data, Kluwer Academic Publishers, Dordrecht, 1991.

[29] Z. Pawlak, Granularity of knowledge, indiscernibility and rough sets, Proc. 1998 IEEE Int. Conf. on Fuzzy Systems, 1998, pp. 106-110.

[30] W. Pedrycz (Ed.) Granular Computing: An Emerging Paradigm, Physica-Verlag, Heidelberg, 2001.

[31] W. Pedrycz, Granular computing with shadowed sets, Proc. 10th Int. Conf. Rough Sets, Fuzzy sets, Data Mining, and Granular Computing, RSFDGrC'05, LNAI 3641, Springer, Berlin, 2005, pp. 23-32.

[32] W. Pedrycz, A. Bargiela, Granular clustering: a granular signature of data, IEEE Transactions on Systems, Man, and Cybernetics, Part B 32 (2002) 212-224.

[33] J.F. Peters, Z. Pawlak, A. Skowron, A rough set approach to measuring information granules, Proc. Int. Conf. Computer Software and Applications, COMPSAC'02, 2002, pp. 1135-1139.

[34] T. Poggio, S. Smale, The mathematics of learning: dealing with data, Notices of AMS 50 (2003) 537-544.

[35] L. Polkowski, A. Skowron, Towards adaptive calculus of granules, Proc. 1998 IEEE Int. Conf. on Fuzzy Systems, 1998, pp. 111-116. 
[36] S.N. Salthe, Evolving Hierarchical Systems, Their Structure and Representation, Columbia University Press, New York, 1985.

[37] H.A. Simon, The organization of complex systems, in: H.H. Pattee (Ed.) Hierarchy Theory, The Challenge of Complex Systems, George Braziller, New York, 1963, pp. 1-27.

[38] M. Sipser, Introduction to the Theory of Computation, Second Edition, Thomson Course Technology, 2006.

[39] A. Skowron, Toward intelligent systems: calculi of information granules, Bulletin of Int. Rough Set Society 5 (2001) 9-30.

[40] A. Skowron, P. Synak, Hierarchical information maps, Proc. 10th Int. Conf. Rough Sets, Fuzzy sets, Data Mining, and Granular Computing, RSFDGrC'05, LNAI 3641, Springer, Berlin, 2005, pp. 622-631.

[41] A. Skowron, J. Stepaniuk, Towards discovery of information granules, Proc. European Conf. on Principles and Practice of Knowledge Discovery in Databases, PKDD’99, LNCS 1704, Springer, Berlin, 1999, pp. 542-547.

[42] A. Skowron, J. Stepaniuk, Information granules: towards foundations of granular computing, Int. Journal of Intelligent Systems 16 (2001) 57-85.

[43] S. Tsumoto, T.Y. Lin, J.F. Peters, Foundations of data mining via granular and rough computing, Proc. 26th Int. Conf. Computer Software and Applications, COMPSAC'02, 2002, pp. 1123-1125.

[44] L.L. Whyte, A.G. Wilson, D. Wilson (Eds.) Hierarchical Structures, American Elsevier Publishing Company, Inc., New York, 1969.

[45] N. Wirth, Program development by stepwise refinement, Communications of ACM 14 (1971) 221-227.

[46] J.T. Yao, Y.Y. Yao, Induction of classification rules by granular computing, Proc. 3rd Int. Conf. on Rough Sets and Current Trends in Computing, RSCTC'02, LNAI 2475, Springer, Berlin, 2002, pp. 331-338. 
[47] Y.Y. Yao, Granular computing using neighborhood systems, in: R. Roy, T. Furuhashi, P.K. Chawdhry (Eds.) Advances in Soft Computing: Engineering Design and Manufacturing, Springer, London, 1999, pp. 539553.

[48] Y.Y. Yao, Granular computing: basic issues and possible solutions, Proc. 5th Joint Conf. on Information Sciences, 2000, pp. 186-189.

[49] Y.Y. Yao, Information granulation and rough set approximation, Int. J. of Intelligent Systems 16 (2001) 87-104.

[50] Y.Y. Yao, Information granulation and approximation in a decisiontheoretical model of rough sets, in: S.K. Pal, L. Polkowski, A. Skowron (Eds.) Rough-Neural Computing: Techniques for Computing with Words, Springer, Berlin, 2003, pp. 491-518.

[51] Y.Y. Yao, A partition model of granular computing, Transactions on Rough Sets 1 (2004) 232-253.

[52] Y.Y. Yao, Granular computing, Computer Science (Ji Suan Ji Ke Xue) 31 (2004) 1-5.

[53] Y.Y. Yao, Perspectives of granular computing, Proc. 2005 IEEE Int. Conf. on Granular Computing, 2005, pp. 85-90.

[54] Y.Y. Yao, Three perspectives of granular computing, Journal of Nanchang Institute of Technology 25 (2006) 16-21.

[55] Y.Y. Yao, Granular computing for data mining, Proc. SPIE Conf. on Data Mining,Intrusion Detection, Information Assurance, and Data Networks Security, 2006, pp. 1-12, (paper no. 624105).

[56] L.A. Zadeh, Towards a theory of fuzzy information granulation and its centrality in human reasoning and fuzzy logic, Fuzzy Sets and Systems 19 (1997) 111-127.

[57] L.A. Zadeh, Some reflections on soft computing, granular computing and their roles in the conception, design and utilization of information/intelligent systems, Soft Computing 2 (1998) 23-25.

[58] B. Zhang, L. Zhang, Theory and Applications of Problem Solving, North-Holland, Amsterdam, 1992. 
[59] L. Zhang, B. Zhang, The quotient space theory of problem solving, Fundamenta Informatcae 59 (2004) 287-298.

[60] Y.Q. Zhang, M.D. Fraser, R.A. Gagliano, A. Kandel, Granular neural networks for numerical-linguistic data fusion and knowledge discovery, IEEE Transactions on Neural Networks 11 (2000) 658-667.

[61] N. Zhong, Multi-database mining: a granular computing approach, Proc. 5th Joint Conf. on Information Sciences, 2000, pp. 198-201. 